\title{
Application aux crues de la Seine, notamment celles de janvier et février 1955, d'une méthode d'investigation des bassins fluviaux en état de réagir à de nouvelles précipitations.
}

\section{Essai de prévision approchée des caractéristiques} de l'onde de crue à Paris (importance, date de passage)

\author{
A method of investigating river basins \\ in which the river reacts to renewed rainfall, \\ applied to the Seine floods, and in particular \\ to those which occured in January and February 1955
}

PAR P. FONTAINE

INGÉXIEUR DF IA METTEOBOLOGIE

On a recherché des critères simples permettant de détecter suffisamment tot les bassins fluviaux en état de réagir da de nouvelles précipitations. Ces critères, établis en fonction de la courbe des précipitations moyennes décadaires, relative a chaque bassin, et de la « normale 》 correspondante, sont particulièrement significatifs pour les régimes d'écoulement du type pluvialocéanique.

On montre, sur des exemples relatifs an bassin de la Seine, que cette méthode permet, en hiver, d'estimer d'une manière acceptable les dates et l'importance probable de l'onde de crue $\dot{a}$ Paris.

\begin{abstract}
Simple criteria were sought which would make it possible to detect in good time the river basins likely to react to renewed rainfall. These crileriu have been established as a function of a curve for each basin representing mean precipitation over ten-day periods, and of the corresponding "normal" rainfall; they have a particular significance for the "pluvial oceanic" types of flow regime.

By means of examples relating to the Seine catchment area, this method is shown to be adequate for predicting, in the winter, with sufficient accuracy, the dates and maqnitude of floods likely to occur in Paris.
\end{abstract}

Les deux crues rćcentes de la Seine à Paris, celle du 23 janvier et celle du 14 février 1955 , ont permis d'expérimenter à nouveau une méthode très simple de détection des bassins fluviaux en état de réagir à de nouvelles précipitations.

Cette méthode, qui a été exposée dans la Revue de la Société Météorologique de France (numéro de janvier-mars 1955), est utilisće depuis plus de deux ans par le Service de Prévisions de la Météorologie, dans le cadre de ses rapports avec les Services d'Annonce des Crues.

Elle a donné une fois encore des résultats satisfaisants, les deux crues récentes ayant été pressenties respectivement douze jours et dix jours avant leur réalisation. 
Mais un examen plus approfondi de la méthode a suggéré la possibilité de prévoir, avec des délais appréciables et des approximations convenables, le jour de passage et l'importance de l'onde de crue à Paris, ce qu'a confirmé l'application de la méthode à des crues antérieures. C'est ce que nous nous proposons d'exposer après un rappel succinct de la méthode utilisée.
I. - Rappel succint de la méthode utilisée pour détecter les bassins fluviaux en état de réagir à de nouvelles précipitations.

Dans son application à chaque bassin, cette méthode repose essentiellement sur l'examen de la courbe des précipitations décadaires, dont cha-

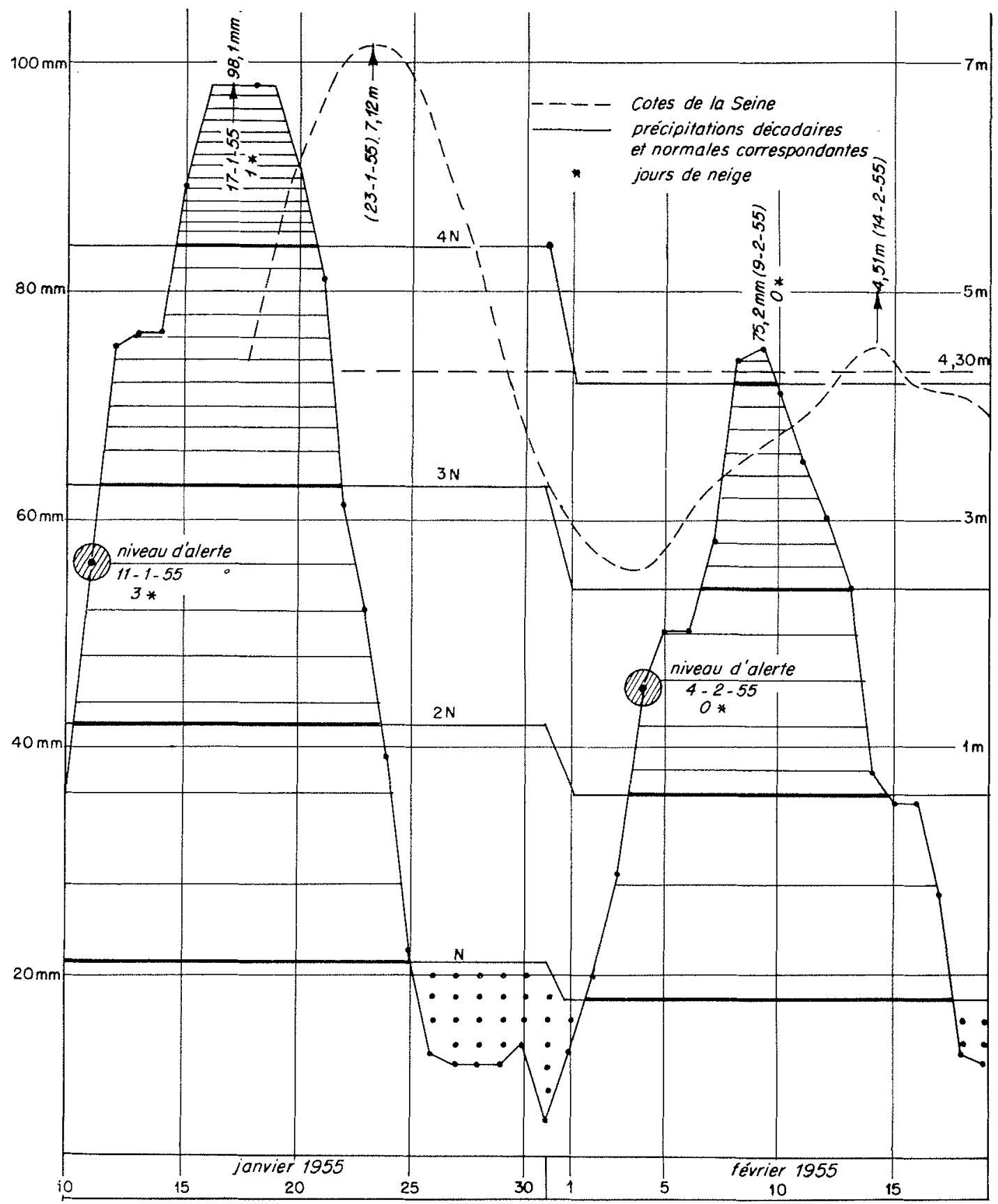

Fig. 1. - Crues à Paris (Pont d'Austerlitz) du 23 janvier $1955(7,12 \mathrm{~m})$ et du 14 f'évrier $1955(4,51 \mathrm{~m})$.

Stations pluviométriques utilisées :

Seine: Romilly-sur-Seine, Châtillon-sur-Seinc.

Marne : Saint-Dizier, Langres.
Yonne: Auxerre, Château-Chinon.

Région parisienne: Paris-Le Bourget. 
que poinl représente la valeur des précipitations du jour, augmentée de celle des neuf jours précédents, étant entendu que les précipitations cruotidiennes résultent de la moyenne d'un certain nombre d'observations pluviométriques représentatives ( $\left.{ }^{\star}\right)$.

La comparaison de cette courbe avec la normale correspondante, égale au tiers de la normale mensuelle, calculée à partir des données des mêmes stations pluviométriques, a permis de définir expérimentalement le niveau pluviométrique à partir duquel on peut envisager la réaction d'un bassin lorsque le régime pluvieux en cours vient à se poursuivre.

On a constaté que ce niveau critique, que nous symboliserons par $2 \mathrm{~N}$, est égal au double de la normale décadaire pour tous les bassins. Seuls diffèrent les «temps de réaction », liés à la structure particulière de chaque bassin.

Dans son application pratique, la méthode d'investigation ainsi définie nécessite évidemment certains ajustements, en fonction des variations saisonnières des débits et des effets de fonte ou de rétention nivale.

Sans entrer plus dans le détail de la méthode, examinons quels enseignements peut apporter son application aux crues récentes de la Seine à Paris, et à un certain nombre de crues antérieures.

\section{II. - Application de la méthode des préci- pitations moyennes décadaires aux crues de la Seine à Paris, observées en janvier et en février 1955.}

Sur le graphique $n^{0} 1$ sont représentées la courbe des précipitations moyennes décadaires pour la période du 10 janvier au 19 février 1955, et celle résultant des cotes successives de la Seine à Paris-Austerlitz pendant la même période.

La comparaison des deux courbes fait apparaitre que le maximum de la crue en janvier (le 23) s'est produit douze jours après la date à laquelle la valeur $2 \mathrm{~N}$ a été atteinte (le 11) et six jours après le maximum pluviométrique décadaire du 17 janvier.

Pour la crue, moins importante, de février (le 14), les déphasages par rapport aux mêmes ćléments sont respectivement de dix jours et de cinq jours. Enfin apparaît aussi un rapport assez étroit entre l'importance de chaque crue et celle du maximum pluviométrique décadaire correspondant.

(*) Pour le bassin de la Seine (amont du confluent de l'Oise) ont été retenues les stations d'Anxerre et de Château-Chinon pour l'Yonne, de Romilly et de Châtillon pour la Seine, de Saint-Dizier et de Langres pour la Marne et de Paris-Le Bonrget pour la région parisienne.
Ces constatations, assez semblables, faites sur deux cas particuliers, ont conduit à penser qu'elles pouvaient avoir un caractère plus général, ce qu'il convenait évidemment de vérifier sur d'autres crues observées dans le passé.

\section{III. - Application de la méthode des pré- cipitations moyennes décadaires à un certain nombre de crues de la Seine, à Paris, observées depuis 1910.}

\section{a) Remarques préliminaires}

Avant d'exposer les résultats obtenus par l'application de la méthode des précipitations moyennes décadaires à un certain nombre de crues, observées à Paris depuis 1910, il est nécessaire de préciser qu'il n'a pas toujours été possible, pour des causes diverses, d'utiliser un réseau pluviométrique ahsolument identique à celui actuellement en service.

Mais dans l'ensemble, pour les neuí crues supplémentaires examinées, les modifications apportées à ce réseau ont été peu importantes.

Par ailleurs, il a été indispensable de tenir compte des effets de fonte ou de rétention nivale sur le comportement des crues à Paris.

C'est ainsi qu'il a été admis que le nombre de jours de neige observés au sein d'une période pluvio-neigeuse affectant le cours moyen et supérieur de la Seine et de ses affluents retardait d'autant le passage de l'onde de crue à Paris, ce qui a conduit à introduire la notion de date théorique pour les deux points caractéristiçues de la courbe des précipitations moyennes décadaires, à savoir ceux correspondant à la valeur $2 \mathrm{~N}$ et au maximum.

Ces dates théoriques sont obtenues par la simple addition aux dates réelles du nombre de jours de neige observés au cours de la décade pluvioneigeuse correspondante.

Quaut à l'importance des maxima décadaires de précipitations, exprimée en millimètres d'eau, il est également nécessaire de la ramencr à ses justes proportions lorsque la décade correspondante vient à se terminer par des jours de neige sans effel jommédiat sur la crue en cours, ou de la surestimer, au contrire, si les pluies de la décade correspondante provoquent la disparition d'une couche de neige résultant de précipitations solides antérieures à cette décade, mais dont on peut estimer la valeur en eau.

Ces divers ajustements, d'ailleurs assez approximatifs, ont permis de rendre comparables les résultats obtenus par l'application de la méthode des précipitations moyennes décadaires à un certain nombre de crues engendrées par des régimes de précipitations plus ou moins complexes, 


\section{b) Résultats obtenus}

Le détail de l'analyse, par cette méthode, des deux crues récentes et de neuf autres crues s'échelonnant jusqu'à l'année 1910, ne peut entrer dans le cadre de cet exposé, ce qui conduit à examiner dès maintenant les résultats obtenus, résumés dans les tableaux I et II.

Sur le tableau I, on peut constater que l'onde de crue à Paris peut être attendue en moyenne dix jours après la date correspondant à la valeur $2 \mathrm{~N}$ des précipitations moyennes décadaires et cinq jours après la date à laquelle celles-ci atteignent leur valeur maximum.

Rappelons que les dates relatives à ces deux valeurs particulières sont les dates théoriques qui ont été définies précédemment. Dates réelles et dates théoriques coïncident évidemment lorsque la crue examinée résulte uniquement de précipitations liquides.

TABLEAU I

DATE DU PASSAGE DE L'ONDE DE CRUE A PARIS

\begin{tabular}{|c|c|c|c|c|c|c|c|c|}
\hline \multicolumn{5}{|c|}{$\begin{array}{l}\text { I. - D'APRìs LA DATE, DE LA VALEUR } 2 \mathrm{~N}\left({ }^{*}\right) \\
\text { DES PRÉCIPITATIONS MOYENNES DÉCADAIRES }\end{array}$} & \multicolumn{4}{|c|}{$\begin{array}{l}\text { II. - D'APRÉs LA DATE, DU MAXINUM } \\
\text { DES PRÉCIPITATIONS MOYENNES DÉGADAIRES }\end{array}$} \\
\hline $\begin{array}{l}\text { Crues à } \\
\text { Paris-Austz } \\
- \\
\text { Date du } \\
\text { maximum }\end{array}$ & $\mid \begin{array}{c}\text { Précipitations } \\
\text { décadaires } \\
\text { Valeur } \geqslant 2 \mathrm{~N} \\
- \\
\text { Date } \\
\text { réelle }\end{array}$ & $\begin{array}{c}\text { Nombre } \\
\text { de jours } \\
\text { de neige } \\
\text { observés } \\
\text { dans la } \\
\text { décade } \\
\text { corres- } \\
\text { pondante }\end{array}$ & $\left|\begin{array}{c}\text { Précipitations } \\
\text { décadaires } \\
\text { Valeur } \geqslant 2 \mathrm{~N} \\
\text { Date } \\
\text { théorique }\end{array}\right|$ & $\begin{array}{l}\text { Déphasages } \\
\text { entre } 1 \text { et } \mathrm{H} \\
- \\
\text { Nombre } \\
\text { de jours }\end{array}$ & $\mid \begin{array}{c}\text { Précipitations } \\
\text { décadaires } \\
\text { Maximum } \\
- \\
\text { Date } \\
\text { réelle }\end{array}$ & $\begin{array}{c}\text { Nombre } \\
\text { de jours } \\
\text { de neige } \\
\text { observés } \\
\text { dans la } \\
\text { décade } \\
\text { corres- } \\
\text { pondante }\end{array}$ & $\mid \begin{array}{c}\text { Précipitations } \\
\text { décadaires } \\
\text { Maximum } \\
- \\
\text { Date } \\
\text { théorique }\end{array}$ & $\begin{array}{l}\text { Déphasages } \\
\text { entre } 1 \text { et } 8 \\
- \\
\text { Nombre } \\
\text { de jours }\end{array}$ \\
\hline $\begin{array}{l}29 \text { janvier } \\
1910\end{array}$ & $\begin{array}{c}18 \text { janvier } \\
1910\end{array}$ & 1 & $\mid \begin{array}{c}19 \text { janvier } \\
1910\end{array}$ & 10 & $\begin{array}{c}21 \text { janvier } \\
1910\end{array}$ & 2 & $\begin{array}{c}23 \text { janvier } \\
1910\end{array}$ & 6 \\
\hline $\begin{array}{r}12 \text { mars } \\
1923\end{array}$ & $\begin{array}{c}26 \text { février } \\
1923\end{array}$ & 1 & $\mid \begin{array}{c}27 \text { février } \\
1923\end{array}$ & 13 & $\begin{array}{r}3 \text { mars } \\
1923\end{array}$ & 1 & $\begin{array}{r}4 \text { mars } \\
1923\end{array}$ & 8 \\
\hline $\begin{array}{c}6 \text { janvier } \\
1924\end{array}$ & $\left|\begin{array}{c}22 \text { décembre } \\
1923\end{array}\right|$ & 4 & $\left|\begin{array}{c}26 \text { décembre } \\
1923\end{array}\right|$ & 11 & $\left|\begin{array}{c}28 \text { décembre } \\
1923\end{array}\right|$ & 4 & $\begin{array}{c}\text { 1er janvier } \\
1924\end{array}$ & 5 \\
\hline $\begin{array}{c}2 \text { décembre } \\
1930\end{array}$ & $\left|\begin{array}{c}20 \text { novembre } \\
1930\end{array}\right|$ & 0 & $\left|\begin{array}{c}20 \text { novembre } \\
1930\end{array}\right|$ & 12 & $\left|\begin{array}{c}24 \text { novembre } \\
1930\end{array}\right|$ & 0 & $\begin{array}{c}24 \text { novembre } \\
1930\end{array}$ & 8 \\
\hline $\begin{array}{r}4 \text { mars } \\
1937\end{array}$ & $\begin{array}{c}19 \text { février } \\
1937\end{array}$ & 1 & $\begin{array}{c}20 \text { février } \\
1937\end{array}$ & 12 & $\begin{array}{c}25 \text { février } \\
1937\end{array}$ & 2 & $\begin{array}{c}27 \text { février } \\
1937\end{array}$ & 5 \\
\hline $\begin{array}{c}4 \text { décembre } \\
1944\end{array}$ & $\mid \begin{array}{c}22 \text { novembre } \\
1944\end{array}$ & 1 & $\begin{array}{c}23 \text { novembre } \\
1944\end{array}$ & 11 & $\left|\begin{array}{c}29 \text { novembre } \\
1944\end{array}\right|$ & 1 & $\mid \begin{array}{c}30 \text { novembre } \\
1944\end{array}$ & 4 \\
\hline $\begin{array}{c}16 \text { février } \\
1945\end{array}$ & $\begin{array}{c}5 \text { février } \\
1945\end{array}$ & 4 & $\begin{array}{c}9 \text { février } \\
1945\end{array}$ & 7 & $\begin{array}{c}11 \text { février } \\
1945\end{array}$ & 0 & $\begin{array}{c}11 \text { février } \\
1945\end{array}$ & 5 \\
\hline $\begin{array}{c}5 \text { décembre } \\
1952\end{array}$ & $\left|\begin{array}{c}25 \text { novembre } \\
1952\end{array}\right|$ & 0 & $\left|\begin{array}{c}25 \text { novembre } \\
1952\end{array}\right|$ & 10 & $\begin{array}{c}1^{\mathrm{er}} \text { décembre } \\
1952\end{array}$ & 0 & $\begin{array}{c}1^{\mathrm{er}} \text { décembre } \\
1952\end{array}$ & 4 \\
\hline $\begin{array}{c}31 \text { décembre } \\
1952\end{array}$ & $\mid \begin{array}{c}16 \text { décembre } \\
1952\end{array}$ & 5 & $\left|\begin{array}{c}21 \text { décembre } \\
1952\end{array}\right|$ & 10 & $\mid \begin{array}{c}20 \text { décembre } \\
1952\end{array}$ & $\overline{5}$ & $\mid \begin{array}{c}25 \text { décembre } \\
1952\end{array}$ & 6 \\
\hline $\begin{array}{c}23 \text { janvier } \\
1955\end{array}$ & $\begin{array}{c}11 \text { janvier } \\
1955\end{array}$ & 3 & $\begin{array}{c}14 \text { janvier } \\
1955\end{array}$ & 9 & $\begin{array}{c}17 \text { janvier } \\
1955\end{array}$ & 1 & $\begin{array}{c}18 \text { janvier } \\
1955\end{array}$ & 5 \\
\hline \multirow[t]{2}{*}{$\begin{array}{c}14 \text { février } \\
1955\end{array}$} & $\begin{array}{l}4 \text { février } \\
1955\end{array}$ & 0 & $\begin{array}{c}14 \text { février } \\
1955\end{array}$ & 10 & $\begin{array}{c}9 \text { février } \\
1955\end{array}$ & 0 & $\begin{array}{c}9 \text { février } \\
1955\end{array}$ & 5 \\
\hline & & \multicolumn{2}{|c|}{ Déphasage moyen $\rightarrow$} & 10 jours & \multicolumn{3}{|c|}{ Déphasage moyen $\rightarrow$} & 5 jours \\
\hline
\end{tabular}


TABLEAU II

\begin{tabular}{|c|c|c|c|}
\hline \multicolumn{2}{|c|}{ Crues a Pains-Austerlit\% } & \multicolumn{2}{|c|}{$\begin{array}{l}\text { PRÉCIPITATIONS DÉCADAIRES } \\
\text { Valeur du maximum }\end{array}$} \\
\hline Date & Hauteur & Non corrigé & $\begin{array}{l}\text { Corrigé } \\
\text { des effets } \\
\text { de fonte } \\
\text { ou de } \\
\text { rétention } \\
\text { nivale }\end{array}$ \\
\hline $\begin{array}{c}29 \text { janvier } \\
1910\end{array}$ & $8,63 \mathrm{~m}$ & $117 \mathrm{~mm}$ & $104 \mathrm{~mm}$ \\
\hline $\begin{array}{r}12 \text { mars } \\
1923\end{array}$ & $5,30 \mathrm{~m}$ & $69 \mathrm{~mm}$ & $69 \mathrm{~mm}$ \\
\hline $\begin{array}{l}6 \text { janvier } \\
1924\end{array}$ & $7,32 \mathrm{~m}$ & $99 \mathrm{~mm}$ & $96 \mathrm{~mm}$ \\
\hline $\begin{array}{l}2 \text { décembre } \\
1930\end{array}$ & $6,08 \mathrm{~m}$ & $71 \mathrm{~mm}$ & $71 \mathrm{~mm}$ \\
\hline $\begin{array}{r}4 \text { mars } \\
1937\end{array}$ & $5,21 \mathrm{~m}$ & $69 \mathrm{~mm}$ & $69 \mathrm{~mm}$ \\
\hline $\begin{array}{c}4 \text { décembre } \\
1944\end{array}$ & $6,03 \mathrm{~m}$ & $80 \mathrm{~mm}$ & $80 \mathrm{~mm}$ \\
\hline $\begin{array}{c}16 \text { fèvrier } \\
1945\end{array}$ & $6,85 \mathrm{~m}$ & $51 \mathrm{~mm}$ & $91 \mathrm{~mm}$ \\
\hline $\begin{array}{c}5 \text { décembre } \\
1952\end{array}$ & $3,65 \mathrm{~m}$ & $67 \mathrm{~mm}$ & $67 \mathrm{~mm}$ \\
\hline $\begin{array}{c}31 \text { décembre } \\
1952\end{array}$ & $4,60 \mathrm{~m}$ & $73 \mathrm{~mm}$ & $73 \mathrm{~mm}$ \\
\hline $\begin{array}{c}23 \text { janvier } \\
1955\end{array}$ & $7,12 \mathrm{~m}$ & $98 \mathrm{~mm}$ & $98 \mathrm{~mm}$ \\
\hline $\begin{array}{c}14 \text { février } \\
1955\end{array}$ & $4,51 \mathrm{~m}$ & $75 \mathrm{~mm}$ & $75 \mathrm{~mm}$ \\
\hline
\end{tabular}

Quant aux écarts à ces valeurs moyennes de dix jours et de cinq jours, ils sont compris, dans la proportion de 70 à $80 \%$, dans l'intervalle \pm 1 jour, les écarts de \pm 3 jours étant exceptionnels.

Sur le tableau II, la comparaison de la hauteur de chaque crue, à Paris, avec la valeur du maximum décadaire de précipitation correspondant paraît suggérer qu'il n'existe pas de rapport aussi étroit qu'on pouvait le penser entre ces deux éléments, malgré les ajustements apportés en fonction des effets de fonte ou de rétention nivale.

C'est ainsi, par exemple, que la crue de janvier 1910 , supérieure d'environ $1,50 \mathrm{~m}$ à celle de janvier 1955, ne la dépasse que de $6 \mathrm{~mm}$ pour ce qui concerne la valeur des maxima décadaires de précipitations.
Mais, portées sous forme de graphique (fig. 2), les valeurs du tableau II permettent de construire deux branches de courbes asymptotes vers les fortes valeurs des maxima décadaires de précipitations.

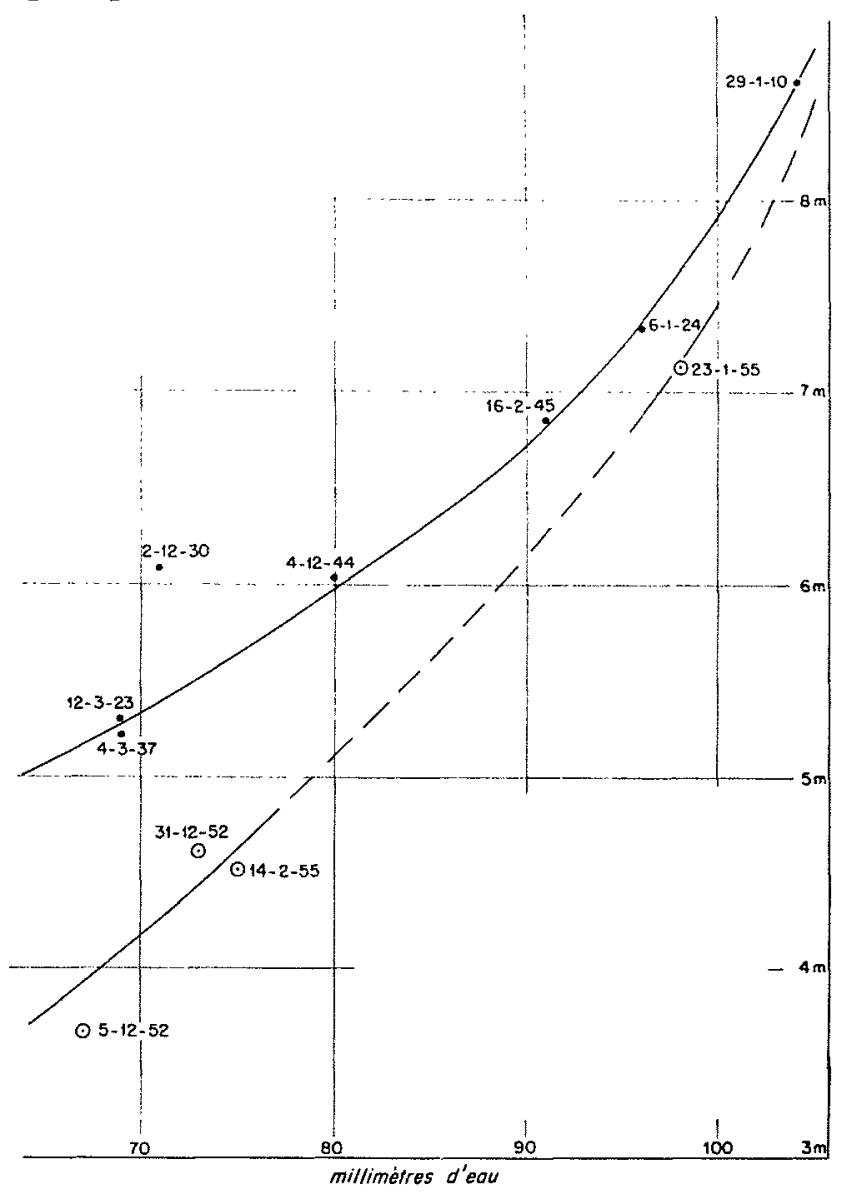

Fig. 2. - Importance des crues à Paris-Austerlitz d'après les maxima décadaires de précipitations correspondants.

La première branche de courbe, relative aux crues postérieures à l'année $\mathbf{1 9 4 5}$, est évidemment assez incomplète dans l'intervalle correspondant à des crues de 5 à 6 mètres, celles-ci ayant fait défaut depuis cette date.

Quant à la seconde branche de courbe, mieux jalonnée, elle présente un point aberrant, celui correspondant à la crue du 2 décembre 1930 .

Malgré tout, ce graphique suggère l'existence d'un rapport assez étroit entre la hauteur de l'onde de crue à Paris et la valeur du maximum décadaire de précipitations correspondant.

Il matérialise également l'atténuation des crues à Paris depuis 1945, par l'effet des travaux entrepris dans ce sens sur le bassin de la Seine. On peut remarquer toutefois que cette atténuation est inversement proportionnelle à l'importance des précipitations. En effet, si pour des maxima décadaires de précipitations de $70 \mathrm{~mm}$, l'abaissement de la crue est d'environ 1 mètre, 
il semble ne devoir guère dépasser $50 \mathrm{~cm}$ pour des maxima décadaires de précipitations atteignant $100 \mathrm{~mm}$.

\section{IV. - Conclusions.}

Cette méthode ne peut certainement pas entrer en concurrence avec celle utilisée par les Services d'annonce des crues, qui recherchent nécessairement une plus grande précision.

Notre manière de procéder néglige notamment certains autres facteurs, tels que le degré de saturation des terrains, la coïncidence des maxima de la Marne et de la Seine, le comportement de l'Oise, etc...

Mais, pour les météorologistes, exercant lcur activité dans de nombreux autres domaines, cette méthode présente un certain intérêt du fait de sa rapidité d'utilisation et des possibilités qu'elle réserve pour le moment où le problème de la prévision quantitative des précipitations, à courte et moyenne échéance, sera résolu.

\section{I S C U S S I O N}

présiden't: M. HUpNeR

Sur la demande de M. le Président, M. ARLeri indique que la période décadaire a été choisie par tâtonnement. M. BaBINET pense que la période optimum doit être du mème ordre de grandeur que la durée de propagation du flot depuis le haut du bassin, laquelle, pour la Seine, est effectivement de l'ordre d'une dizaine de jours.

M. le Président ajoute que si on appliquait les principes de l'hydrogramme unitaire, dont l'idée paraît être à l'origine de cette méthode, on pourrait perfectionner celle-ci en faisant la moyenne pondérée du ruissellement suivant les surfaces partielles du bassin versant intéressées par les pluviomètres correspondants et suivant la position de ces surfaces vis-à-vis des stations hydrométriques considérées. M. BaBINET s'associe à cette remarque.

M. ARLery pense qu'il serait plus simple de multiplier le nombre de stations pluviométriques que d'affecter celles-ci d'un coefficient. Il ajoute, sur la demande de M. le Président, que la neige est prise en compte dans la méthode sous la forme d'un décalage supplémentaire de l'onde de crue de un ou deux jours, suivant qu'il a neigé une ou deux fois, sans tenir compte de l'importance des chutes de neige.

M. le Président souligne que cette méthode, malgré sa précision encore modeste, permet une prévision à échéance de 10 ou 12 jours : elle s'associe donc à des méthodes sans doute plus précises, mais à plus courte ćchéance, dans les moyens mis à la disposition du Service d'Annonce des Crues.

M. Babinet estime que la conjugaison de cette prévision du temps de la crue avec une prévision quantitative de la hauteur ou du débit de crue est souhaitable quoique assez difficile à réaliser, mais que cette prévision du temps maximum est déjà très intéressante en soi.

M. Babinet se montrant surpris que le facteur $2 \mathrm{~N}$ (double de la précipitation normale) qui annonce la probabilité d'une crue, soit assez variable pour des époques voisines de l'année, M. Arlery indique que la précipitation normale décadaire a été, par approximation, considerce comme le tiers de la précipitation mensuelle normale. Il semble qu'il y aurait intérêt à préciser cette notion de période de référence, en considérant exactement les précipitatons décadaires
M. Lallement précise que, pour la erue de janvier 1955, les pluies s'étant pratiquement arrêtées le lundi 17 janvier, la méthode Bachet avait donné, dès le mardi, pour date du maximum de la crue à Paris (maximum dû aux affluents lents) le lundi 24 janvier alors qu'il s'est produit le dimanche 23 janvier dans l'après-midi. On peut expliquer ce décalage dans le temps par des décalages en hauteur dûs aux travaux qui ont modifié le régime de la Seine depuis la période d'observations qui a été utilisée pour le calcul des réglettes Bachet.

En ce qui concerne la méthode décadaire qui vient d'être exposée, M. LaLlement remarque que si les pluies prévues par la Météorologie après le 16 janvier s'étaient produites, la prévision de la date du maximum, donnée par la méthode décadaire, n'aurait pas été changée alors que le maximum ne se serait en fait réalisé que deux jours après.

M. Olivier-Martin précise que le franchissement de l'étape $2 n$ montre seulement qu'à partir de ce momentlà, il pent $\mathrm{y}$ avoir un phénomène grave; d'autre part, on conçoit que ce phénomène sera d'autant plus éloigné dans le temps que la durée et l'intensité des précipitations augmenteront.

M. BABINET indique qu'en janvier 1955 , les précipilations de la période décadaire, cause immédiate de la crue, étaient en moyenne assez voisines de ce qu'elles étaient dans la période correspondante de 1910; dès lors, la différence constatée dans l'importance des crues respectives de 1955 et de 1910 tient à me différence dans la répartition des précipitations qui les ont provoquées. De plus, en 1955 , la période critique ne fut suivie d'aucune précipitation importante alors qu'en 1910 suivit, trois jours après, une nouvelle période de précipitations.

En 1955, le maximum de la crue à Paris a donc surtout été provorué par les crues des affluents lents, tandis que la crue de 1910 a été causée par la coïncidence au confluent de deux crues engendrées successivement, la premièrc intéressant les affluents lents, la deuxième les affluents immédiats et également ceux de la région du Morvan. D'où l'intérêt, souligné par M. Ie Président, d'introduire des décalages dans l'application de la méthode décadaire. 